Research Article

\title{
Fourier Spectral Method for a Class of Nonlinear Schrödinger Models
}

\author{
Lei Zhang $\mathbb{D}^{\circ}$, Weihua Ou Yang $\mathbb{D}^{\mathbb{D}}$, Xuan Liu $\mathbb{D}^{D}$, and Haidong Qu \\ Department of Mathematics, Hanshan Normal University, Chaozhou 515041, China \\ Correspondence should be addressed to Weihua Ou Yang; ouyang2008@hstc.edu.cn
}

Received 15 March 2021; Revised 29 April 2021; Accepted 23 May 2021; Published 1 July 2021

Academic Editor: Jorge E. Macias-Diaz

Copyright (C) 2021 Lei Zhang et al. This is an open access article distributed under the Creative Commons Attribution License, which permits unrestricted use, distribution, and reproduction in any medium, provided the original work is properly cited.

\begin{abstract}
In this paper, Fourier spectral method combined with modified fourth order exponential time-differencing Runge-Kutta is proposed to solve the nonlinear Schrödinger equation with a source term. The Fourier spectral method is applied to approximate the spatial direction, and fourth order exponential time-differencing Runge-Kutta method is used to discrete temporal direction. The proof of the conservation law of the mass and the energy for the semidiscrete and full-discrete Fourier spectral scheme is given. The error of the semidiscrete Fourier spectral scheme is analyzed in the proper Sobolev space. Finally, several numerical examples are presented to support our analysis.
\end{abstract}

\section{Introduction}

Schrödinger equation, being known as basic assumption of quantum mechanics, is one of the most important equations in quantum mechanics that proposed by Austrian physicist Schrödinger. Known to all, the standard Schrödinger equation is a second-order partial differential equation established by combining the concept of matter wave and wave equation, and it can describe the movement of microparticles. Indeed, each microsystem has a corresponding Schrodinger equation. The specific form of wave function and corresponding energy can be obtained by solving this equation, so as to understand the properties of microsystem. Schrödinger equation shows that in quantum mechanics, particles appear in the form of probability, with uncertainty, and can be ignored at the macroscale.

In recent decades, more and more scholars have been interested in studying Schrödinger equations, and the research on Schrödinger equations can be divided into two categories: integral order (or local) and fractional order (or nonlocal) models. Now, we will make a brief literature review.

(1) Schrödinger Equations of Fractional Order. In 2000, firstly, Laskin [1] extended the fractional concept in quantum physics to construct a fractional path integral, and over these paths, the standard nonlinear Schrödinger equation was generalized to the fractional one. Very recently, many scholars studied the numerical solutions of fractional Schrödinger equations. In 2008, Rida et al. [2] applied the Adomian decomposition method to get the analytic and approximate solutions for different types of fractional differential equations. In 2011, the generalized twodimensional differential transform method was directly applied to solve the coupled Burger's equations by Liu et al. [3]. In 2012, Wei et al. [4] presented an implicit fully discrete local discontinuous Galerkin finite element method for solving the time-fractional Schrödinger equation with Caputo fractional derivative. In 2013, Secchi et al. [5] obtained the solutions to a class of Schrödinger equations by using the fractional Laplacian. In 2014, the Jacobi spectral collocation method was constructed, and the numerical solutions of the time-fractional Schrödinger equation and the space-fractional Schrödinger equation were got by Bhrawy et al. [6]. In literature [7], Wang et al. proposed a linearly implicit conservative difference scheme for the coupled nonlinear Schrödinger equations with space fractional derivative, and she 
proved that the scheme can conserve the mass and energy in the discrete level. In 2015, Wang et al. [8] studied the energy conservative Crank-Nicolson difference scheme for nonlinear Riesz space-fractional Schrödinger equations and gave the proof of mass conservation and energy conservation in the discrete sense. In 2016, Ran et al. [9], under some assumptions, an implicit difference scheme was proposed to solve the strongly coupled nonlinear space fractional Schrödinger equations. In the literature [10-12], the semidiscrete scheme was fully constructed by employing finite element method. In 2020, Wang et al. [13] proposed a preconditioning method for the space fractional coupled nonlinear Schrödinger equations. For other literature on the fractional Schrödinger equations, see [14-16]

(2) Schrödinger Equations of Integral Order. The study of the standard Schrödinger equations has attracted many scholars. In 1995, Edwards et al. [17] presented a numerical treatment to solve the time-independent nonlinear Schrödinger equation with an external potential. In 1999, Chang et al. [18] studied the finite difference schemes for solving the generalized nonlinear Schrödinger equations, and several schemes, including Crank-Nicolson-type schemes, split step Fourier scheme, and pseudo-spectral scheme, were employed for solving three models of the generalized nonlinear Schrödinger equations. In 2003, Bao et al. [19] studied the performance of time-splitting spectral approximations for general nonlinear Schrödinger equations and proved that the discrete scheme was unconditionally stable and conserved in $L_{1}$ norm. In 2005, Wang [20] got the numerical solutions to nonlinear Schrödinger equations by using the split-step finite difference method. In 2017, Dehghan et al. [21] presented a numerical scheme based on radial basis function for solving the highdimensional nonlinear Schrödinger equations using the explicit Runge-Kutta method. In 2019, Ahsan et al. [22] proposed a Haar wavelet collocation method for linear and nonlinear Schrödinger equations, and the stability analysis of this method was given. In 2020, Ban et al. [23] proposed a family of algorithms, which were special implementations of Lubich's Convolution Quadrature, for the numerical solution to the Schrödinger equation. For other literature on the standard Schrödinger equations, see [24-28]

In this paper, we study the numerical solution to the nonlinear Schrödinger equation of the following form:

$$
i u_{t}(x, t)+\alpha \Delta u(x, t)+\beta|u(x, t)|^{2} u(x, t)+\gamma f(x) u(x, t)=0
$$

with the periodic condition

$$
u(x+2 \pi, t)=u(x, t)
$$

and the initial condition

$$
u(x, 0)=u_{0}(x)
$$

where

$$
\Delta u(x, t)=\partial_{x x} u(x, t),(x, t) \in(-\pi, \pi) \times[0, T] .
$$

$i$ is the imaginary number of units and $i^{2}=-1 ; u_{0}(x)$ is a function with a period of $2 \pi ; \alpha, \beta$, and $\gamma$ are the three real numbers.

For this model, two important physical quantities are often considered; these are mass and energy and using the conservation of mass and energy to measure whether the algorithm is effective for this equation. As in [10], we can easily derive the mass and energy conservation

$$
\begin{gathered}
M(t)=M(0), \\
E(t)=E(0),
\end{gathered}
$$

where

$$
\begin{aligned}
M(t)= & \int_{0}^{2 \pi}|u(x, t)|^{2} d x, \\
E(t)= & -\alpha \int_{0}^{2 \pi} u_{x}(x, t) \bar{u}_{x}(x, t) d x \frac{\beta}{2} \int_{0}^{2 \pi}|u(x, t)|^{4} d x \\
& +\gamma \int_{0}^{2 \pi} f(x)|u(x, t)| d x .
\end{aligned}
$$

The rest of this paper is organized as follows. In Section 2, the definitions and properties of the related Sobolev space are introduced; based on this space, we establish a semidiscrete Fourier spectral scheme for model (1), whose solution keeps mass and energy conversation for some function $f(x)$. Then, the error estimation of this semidiscrete scheme is given in the established Sobolev space. Section 3 gives an introduction of modified fourth order exponential time-differencing Runge-Kutta method that is used to discrete the temporal component, and a proof of mass conservation and energy conservation is given in the discrete sense. In Section 4, we carry out some numerical experiments to verify our conclusions. Finally, Section 5 offers some concluding remarks.

\section{Preliminaries}

In this section, based on the semidiscrete Fourier spectral scheme, we prove the mass and energy conversation for this semidiscrete scheme and give the error estimation of the Fourier spectral approximation. First, we introduce the following Sobolev space and some notations.

Let $\Omega=(-\pi, \pi) \subset \mathbb{R}$, and the space $C_{p}^{\infty}$ is defined by

$$
C_{p}^{\infty}=\left\{u \mid u \in C^{\infty}(\Omega), u(x-\pi, t)=u(x+\pi, t), x \in \mathbb{R}\right\} .
$$


Define inner product on $\Omega=(-\pi, \pi)$ :

$$
\langle u(x, t), v(x, t)\rangle_{\Omega}=\int_{-\pi}^{\pi} u(x, t) \bar{v}(x, t) d x
$$

and the norm on $\Omega=(-\pi, \pi)$ :

$$
\|u(x, t)\|=\int_{-\pi}^{\pi} u(x, t) \bar{u}(x, t) d x
$$

where $\bar{v}(x, t)$ is the conjugate of $v(x, t)$. We denote the complete space of $C_{p}^{\infty}$ by $H_{p}^{n}$, which can be characterized in the frequency space under the norm

$$
\|u\|_{n}:=\sum_{m \leq n}\left(\left\|D_{m} u(x, t)\right\|^{2}\right)^{1 / 2},
$$

the seminorm $|u|_{n}$ is defined by

$$
|u|_{n}:=\sum_{0<m \leq n}\left(\left\|D_{m} u(x, t)\right\|^{2}\right)^{1 / 2}
$$

where $D_{m} u(x, t)=\partial^{m} u(x, t) / \partial x^{m}$.

Suppose

$$
S_{N}=\operatorname{span}\left\{e^{i k x},-N \leq k \leq N\right\} .
$$

Then, the orthogonal projection operator $P_{N}: H_{p}^{n} \longrightarrow$ $S_{N}$ is defined by

$$
\left(P_{N} u\right)(x)=\sum_{k=-N}^{N} \widehat{u}_{k} e^{i k x}=u^{N}
$$

where $\widehat{u}_{k}, k=-N, \cdots, N$ are the Fourier coefficients of $u$.

According to the definition of inner product, $\forall v^{N} \in S_{N}$, we define the semidiscrete scheme for model (1):

$$
\begin{gathered}
\left\langle i u_{t}^{N}, v^{N}\right\rangle_{\Omega}+\alpha\left\langle\Delta u^{N}, v^{N}\right\rangle_{\Omega}+\beta\left\langle\left|u^{N}\right|^{2} u^{N}, v^{N}\right\rangle_{\Omega}+\gamma\left\langle f u^{N}, v^{N}\right\rangle_{\Omega}=0 \\
u^{N}(0)=P_{N} u_{0}(x)
\end{gathered}
$$

Usually, when designing a numerical scheme for (14), two important conservation laws often need to be considered: the mass and the total energy of the wave function. In the following lemma, we prove that the semidiscrete Fourier spectral scheme (14) can keep the conservation laws.

Lemma 1. Let $u^{N}(0), u^{N}(x, t) \in H_{P}^{1}$, then the semidiscrete scheme (14) is conservative in the sense

$$
\begin{aligned}
M(t) & =M(0), 0<t<T, \\
E(t) & =E(0), 0<t<T,
\end{aligned}
$$

where

$$
\begin{aligned}
M(t)= & \int_{-\pi}^{\pi}\left|u^{N}(x, t)\right|^{2} d x, \\
E(t)= & -\alpha \int_{-\pi}^{\pi} u_{x}^{N}(x, t) \bar{u}_{x}^{N}(x, t) d x+\frac{\beta}{2} \int_{-\pi}^{\pi}\left|u^{N}(x, t)\right|^{4} d x \\
& +\gamma \int_{-\pi}^{\pi} f(x)\left|u^{N}(x, t)\right|^{2} d x,
\end{aligned}
$$

are the mass and energy of the wave function, respectively.

Proof. Let $v^{N}=u^{N}$ in (14), we have

$$
\left\langle\mathrm{i} u_{t}^{N}, u^{N}\right\rangle_{\Omega}+\alpha\left\langle\Delta u^{N}, u^{N}\right\rangle_{\Omega}+\beta\left\langle\left|u^{N}\right|^{2} u^{N}, u^{N}\right\rangle_{\Omega}+\gamma\left\langle f u^{N}, u^{N}\right\rangle_{\Omega}=0 .
$$

Note that $\alpha, \beta$, and $\gamma$ are three real numbers; thus, taking the imaginary part of the above equation and noting the periodic boundary condition, we get

$$
\begin{gathered}
\operatorname{Im}\left(\alpha\left\langle\Delta u^{N}, u^{N}\right\rangle_{\Omega}\right)=\operatorname{Im}\left(\int_{-\pi}^{\pi} u_{x}^{N} \bar{u}_{x}^{N}\right)=0, \\
\operatorname{Im}\left(\beta\left\langle\left|u^{N}\right|^{2} u^{N}, u^{N}\right\rangle_{\Omega}\right)=\operatorname{Im}\left(\beta\left|u^{N}\right|^{2}\left\langle u^{N}, u^{N}\right\rangle_{\Omega}\right)=0, \\
\operatorname{Im}\left(\gamma\left\langle f u^{N}, u^{N}\right\rangle_{\Omega}\right)=0
\end{gathered}
$$

which indicates

$$
\left\langle u_{t}^{N}, u^{N}\right\rangle_{\Omega}=\frac{1}{2} \frac{d}{d t}\left(\left\langle u^{N}, u^{N}\right\rangle_{\Omega}\right)=\frac{1}{2} \frac{d}{d t}(M(t))=0 .
$$

Next, we prove the semidiscrete scheme (14) can keep energy conservation. Let $v^{N}=u_{t}^{N}$ in (14), we have

$$
\left\langle i u_{t}^{N}, u_{t}^{N}\right\rangle_{\Omega}+\alpha\left\langle\Delta u^{N}, u_{t}^{N}\right\rangle_{\Omega}+\beta\left\langle\left|u^{N}\right|^{2} u^{N}, u_{t}^{N}\right\rangle_{\Omega}+\gamma\left\langle f u^{N}, u_{t}^{N}\right\rangle_{\Omega}=0 .
$$

Taking the real part of the above equation and noting the periodic boundary condition, we have

$$
\begin{gathered}
\operatorname{Re}\left(\left\langle i u_{t}^{N}, u_{t}^{N}\right\rangle_{\Omega}\right)=0, \\
\operatorname{Re}\left(\alpha\left\langle\Delta u^{N}, u_{t}^{N}\right\rangle_{\Omega}\right)=\operatorname{Re}\left(\alpha\left\langle u_{x x}^{N}, u_{t}^{N}\right\rangle_{\Omega}\right) \\
=\alpha \operatorname{Re}\left(\int_{-\pi}^{\pi} u_{x x}^{N} \bar{u}_{t}^{N} d x\right) \\
=-\alpha \operatorname{Re}\left(\int_{-\pi}^{\pi} u_{x}^{N} \bar{u}_{x, t}^{N} d x\right) \\
=-\frac{\alpha}{2} \frac{d}{d t} \int_{-\pi}^{\pi} u_{x}^{N} \bar{u}_{x}^{N} d x .
\end{gathered}
$$


Computing the real part of $\beta\left\langle\left|u^{N}\right|^{2} u^{N}, u_{t}^{N}\right\rangle_{\Omega}$, we have

$$
\begin{aligned}
\operatorname{Re} & \left(\beta\left\langle\left|u^{N}\right|^{2} u^{N}, u_{t}^{N}\right\rangle_{\Omega}\right), \\
= & \operatorname{Re}\left(\beta \int_{-\pi}^{\pi}\left(\left|u^{N}\right|^{2} u^{N} \bar{u}_{t}^{N} d x\right)\right) \\
= & \operatorname{Re}\left(\beta \int_{-\pi}^{\pi}\left(u^{N} \bar{u}^{N} u^{N} \bar{u}_{t}^{N}\right) d x\right) \\
= & \frac{\beta}{4} \frac{d}{d t} \int_{-\pi}^{\pi}\left(u^{N} \bar{u}^{N} u^{N} \bar{u}^{N}\right) d x \\
= & \frac{\beta}{4} \frac{d}{d t} \int_{-\pi}^{\pi}\left|u^{N}\right|^{4} d x .
\end{aligned}
$$

Computing the real part of $\gamma\left\langle f u^{N}, u_{t}^{N}\right\rangle_{\Omega}$, we have

$$
\operatorname{Re}\left(\gamma\left\langle f u^{N}, u_{t}^{N}\right\rangle_{\Omega}\right)=\frac{\gamma}{2} \frac{d}{d t} \int_{-\pi}^{\pi} f\left|u^{N}\right|^{2} d x
$$

It follows from equations (22), (23), (24), and (25); we get the energy conservation law. This completes the proof.

Next, we analyze the existence and uniqueness of solution for the semidiscrete scheme (14). We need the following lemma.

Lemma 2. Let $u^{N}$ is the solution for the semidiscrete scheme (14); then, there exists a positive constant $C$, such that

$$
\max \left\{\left\|u^{N}\right\|_{1},\left\|u^{N}\right\|_{\infty}\right\} \leq C
$$

Proof. From Lemma 1, we have

$$
\begin{aligned}
& \left\|u^{N}(t)\right\|_{0}^{2}=\left\|u^{N}(0)\right\|_{0}^{2}=\left\|P_{N} u_{0}(x)\right\|_{0}^{2} \leq\left\|u_{0}\right\|_{0}^{2}=C, \\
& \left\|u^{N}(t)\right\|_{0}^{2}+\frac{\beta}{2} \int_{-\pi}^{\pi}\left|u^{N}(x, t)\right|^{4} d x \\
& \quad\left\|u^{N}(0)\right\|_{0}^{2}+\frac{\beta}{2} \int_{-\pi}^{\pi}\left|u^{N}(x, 0)\right|^{4} d x \\
& \leq\left\|u^{N}(0)\right\|_{0}^{2}+\frac{|\beta|}{2} \int_{-\pi}^{\pi} \max \left\{\left|u^{N}(x, 0)\right|^{4}\right\} d x \\
& \leq\left\|u^{N}(0)\right\|_{0}^{2}+|\beta| \pi\left\|u^{N}(x, 0)\right\|_{\infty}^{4} \\
& \leq\left\|u^{N}(0)\right\|_{0}^{2}+C|\beta| \pi\left\|u^{N}(x, 0)\right\|_{1}^{4}=C .
\end{aligned}
$$

(27) and (28) lead to

$$
\left\|u^{N}(t)\right\|_{1}^{2}=\left\|u^{N}(t)\right\|_{0}^{2}+\left\|u_{x}^{N}(t)\right\|_{0}^{2} \leq C .
$$

Finally, using Sobolev's embedding theorem, we obtain

$$
\left\|u^{N}(t)\right\|_{\infty}^{2} \leq C
$$

which implies both $\left\|u^{N}(t)\right\|_{1}$ and $\left\|u^{N}(t)\right\|_{\infty}$ are bounded.
Therefore

$$
\max \left\{\left\|u^{N}\right\|_{1},\left\|u^{N}\right\|_{\infty}\right\} \leq C
$$

This completes the proof.

The semidiscrete scheme (14) is a first order nonlinear ordinary differential equation with respect to time $t$ on a finite interval; using Lemma 2 and Picard's theorem, we obtain the following theorem.

Theorem 3. Let the solution $u \in L^{2}\left((0, T), H_{P}^{1}(0,2 \pi)\right), u_{0} \in$ $H_{P}^{1}(0,2 \pi)$, then the semi-discrete scheme (14) has unique solution $u^{N}$ in $S_{N}$.

Proof. The proof is omit.

In the following theorem, we will give the error estimation of the Fourier spectral method to solve problem (1). For convenience, we still use $\eta \leq \xi$ to denote that there exists a positive constant $C$, which is independent of $N, \eta$, and $\xi$, such that $\eta \leq C \xi$.

Theorem 4. Let $u$ is the solution of $(1) ; u^{N}$ is obtained from the semidiscrete scheme (14); then, we have

$$
\left\|u(t)-u^{N}(t)\right\|^{2} \leq N^{2 \mu-2} .
$$

Proof. Let $e^{N}(t)=u^{N}(t)-P_{N} u(t)$, with $u_{N}(t)$ in (14) replaced by $P_{N} u(t)$, for all $v^{N} \in S_{N}$, we have

$$
\begin{aligned}
& i\left\langle e^{N, t}(t), v^{N}\right\rangle_{\Omega}+\alpha\left\langle\Delta e^{N}(t), v^{N}\right\rangle_{\Omega} \\
& \quad+\beta\left\langle\left|u^{N}(t)\right|^{2} u^{N}(t)-\left|P_{N} u(t)\right|^{2} P_{N} u(t), v^{N}\right\rangle_{\Omega} \\
& \quad+\gamma\left\langle f e^{N}(t), v^{N}\right\rangle_{\Omega}=0 .
\end{aligned}
$$

Note that $\left\langle u(t)-P_{N} u(t), v^{N}\right\rangle_{\Omega}=0, \forall v^{N} \in S_{N}$, by setting $v^{N}=e^{N}(t)$, and taking the imaginary part of (33), we obtain

$$
\frac{\alpha}{2} \frac{d}{d t}\left\langle e^{N}(t), e^{N}(t)\right\rangle_{\Omega}+\beta \operatorname{Im}\left(\left\langle|u(t)|^{2} u(t)-\left|u^{N}(t)\right|^{2} u^{N}(t), e^{N}(t)\right\rangle_{\Omega}\right)=0 .
$$

Applying the Schwarz inequality to the above equation, we get

$$
\begin{aligned}
\frac{|\alpha|}{2} \frac{d}{d t}\left\|e^{N}(t)\right\|^{2} & \leq\left\||\beta|\left(|u(t)|^{2} u(t)-\left|u^{N}(t)\right|^{2} u_{N}(t)\right)\right\| \cdot\left\|e^{N}(t)\right\| \\
& \leq \frac{1}{2}\left\||\beta|\left(|u(t)|^{2} u(t)-\left|u^{N}(t)\right|^{2} u^{N}(t)\right)\right\|^{2}+\frac{1}{2}\left\|e^{N}(t)\right\|^{2} .
\end{aligned}
$$


We note that

$$
\begin{aligned}
|\beta| & \left(|u(t)|^{2} u(t)-\left|u^{N}(t)\right|^{2} u^{N}(t)\right) \\
= & |\beta||u(t)|^{2}\left(u(t)-u^{N}(t)\right)+|\beta| u^{N}(t) \\
& \cdot\left\{\bar{u}(t) u(t)-u(t) \bar{u}^{N}(t)+u(t) \bar{u}^{N}(t)-\bar{u}^{N}(t) u^{N}(t)\right\} \\
= & |\beta|\left\{|u(t)|^{2}\left(u(t)-u^{N}(t)\right)+u^{N}(t) u(t)(\bar{u}(t)\right. \\
& \left.\left.-\bar{u}^{N}(t)\right)+\left|u^{N}(t)\right|^{2}\left(u(t)-u^{N}(t)\right)\right\} .
\end{aligned}
$$

Then, the factor $\left\||\beta|\left(|u(t)|^{2} u(t)-\left|u^{N}(t)\right|^{2} u^{N}(t)\right)\right\|^{2}$ is bounded by

$$
\begin{aligned}
& \left\||\beta|\left(|u(t)|^{2} u(t)-\left|u^{N}(t)\right|^{2} u^{N}(t)\right)\right\| \leq\left\|u(t)-u^{N}(t)\right\| \\
& \quad \leq\left\|u(t)-P_{N} u(t)\right\|+\left\|e^{N}(t)\right\| .
\end{aligned}
$$

According to equations (35) and (37) and $\left(u-P_{N} u, e^{N}\right)$ $=0$, we arrive at

$$
\frac{d}{d t}\left\|e^{N}(t)\right\|^{2} \leq\left\|u(t)-P_{N} u(t)\right\|^{2}+\left\|e^{N}(t)\right\|^{2},
$$

which in accordance with Gronwall inequality, we have

$$
\left\|e^{N}(t)\right\| \leq \int_{0}^{T}\left\|u(\tau)-P_{N} u(\tau)\right\|^{2} d \tau
$$

Noting that for all $0<\mu<1$, there exists a positive constant $C$, such that

$$
\left\|u(t)-P_{N} u(t)\right\|_{\mu} \leq C N^{\mu-1}|u|_{1}
$$

Finally, a combination of the above estimates leads to

$$
\begin{aligned}
\left\|u(t)-u^{N}(t)\right\|^{2} & \leq\left\|u(t)-P_{N} u(t)\right\|^{2}+\left\|P_{N} u(t)-u^{N}(t)\right\|^{2} \\
& \leq N^{2 \mu-2}|u(t)|_{1}^{2}+\int_{0}^{T}\left\|u(\tau)-P_{N} u(\tau)\right\|^{2} d \tau \\
& \leq N^{2 \mu-2}|u(t)|_{1}^{2}+\left.N^{2 \mu-2}\right|_{0} ^{T}|u(\tau)|_{1}^{2} d \tau \\
& \leq N^{2 \mu-2}\left(|u(t)|_{1}^{2}+\int_{0}^{T}|u(\tau)|_{1}^{2} d \tau\right) \leq N^{2 \mu-2}
\end{aligned}
$$

This completes the proof.

\section{The Full-Discrete Fourier Spectral Scheme and Its Conservation}

In this section, we first introduce the full-discrete Fourier spectral scheme for (1) and then give the proof of its conservation properties.
3.1. The Full-Discrete Fourier Spectral Scheme. Due to the periodicity of the solution, it suffices to compute the model in the interval $\Omega=[-\pi, \pi]$. We choose $N$ interpolation points $x^{N}=\left(x_{1}, x_{2}, \cdots, x_{N}\right)$, and $x_{j}=2 \pi j / N, \quad-N / 2 \leq j \leq N / 2-1$. Then, the semidiscrete scheme (14) can be discretized in space by seeking the approximate solution

$$
u\left(x_{j}\right)=\sum_{|k|_{\infty} \leq N / 2} \tilde{u}_{k} e^{i k x_{j}}, t>0
$$

where

$$
\tilde{u}_{k}=\frac{C_{k}}{N} \sum_{j=1}^{n} u\left(x_{j}\right) e^{i k x_{j}}
$$

is the $k$ th discrete Fourier coefficient, $C_{k}=1$ for $|k|<N / 2$, and $C_{k}=2$ for $|k|=N / 2$. Thus, for each frequency $k$, we have

$$
\left\{\begin{array}{l}
i \frac{\partial \tilde{u}_{k}}{\partial t}+\alpha\left(k^{2}\right) \tilde{u}_{k} \\
+\beta \mathscr{F}\left(\left|\mathscr{F}^{-1}\left(\tilde{u}_{k}\right)\right|^{2} \mathscr{F}^{-1}\left(\tilde{u}_{k}\right)\right)+\gamma \mathscr{F}\left(f\left(\mathscr{F}^{-1}\left(\tilde{u}_{k}\right)\right)\right)=0, \\
\tilde{u}(x, 0)=\mathscr{F}\left(u_{0}\right) .
\end{array}\right.
$$

Let $h_{1}, h_{2}, \cdots, h_{Q}$ be the time steps, satisfying

$$
\begin{aligned}
\sum_{q=1}^{Q} h_{q}= & T, \\
t_{q}= & \sum_{j=1}^{q} h_{j}, \\
L= & i \alpha\left(k^{2}\right), \\
F\left(\tilde{u}_{k}, t\right)= & \mathrm{i} \beta \mathscr{F}\left(\left|\mathscr{F}^{-1}\left(\tilde{u}_{k}\right)\right|^{2} \mathscr{F}^{-1}\left(\tilde{u}_{k}\right)\right) \\
& +\mathrm{i} \gamma \mathscr{F}\left(f\left(\mathscr{F}^{-1}\left(\tilde{u}_{k}\right)\right)\right),
\end{aligned}
$$

then (44) is equivalent to

$\tilde{u}_{k}\left(t_{q}+h_{q+1}\right)=e^{L h_{q+1}} \tilde{u}_{k}\left(t_{q}\right)+e^{L h_{q+1}} \int_{0}^{h_{q+1}} e^{-L s} F\left(\tilde{u}_{k}\left(t_{q}+s\right), t_{q}+s\right) d s$.

More detail about this equation can be find in $[29,30]$. Now, let $\tilde{u}_{k, q}=\tilde{u}_{k}\left(t_{q}\right)$ and using modified fourth order exponential time-differencing Runge-Kutta (ETDRK4) method to approximate the integral equation (46), we have 


$$
\left\{\begin{array}{l}
\lambda_{q}=e^{\frac{L h_{q+1}}{2}} \tilde{u}_{k, q}+L^{-1}\left(e^{\frac{L h_{q+1}}{2}}-I\right) F\left(\tilde{u}_{k, q}, t_{q}\right), \\
\chi_{q}=e^{\frac{L h_{q+1}}{2}} \tilde{u}_{k, q}+L^{-1}\left(e^{\frac{L h_{q+1}}{2}}-I\right) F\left(\lambda_{q}, t_{q}+\frac{h_{q+1}}{2}\right), \\
\nu_{q}=e^{\frac{L h_{q+1}}{2}} \lambda_{q}+L^{-1}\left(e^{\frac{L h_{q+1}}{2}}-I\right) . \\
\left(2 F\left(\chi_{q}, t_{q}+\frac{h_{q+1}}{2}\right)-F\left(\tilde{u}_{k, q}, t_{q}\right)\right) \\
\tilde{u}_{k, q+1}=e^{L h_{q+1}} \tilde{u}_{k, q}+h_{q+1}\left\{\theta_{1} F\left(\tilde{u}_{k, q}, t_{q}\right)\right. \\
+2 \theta_{2}\left(F\left(\lambda_{q}, t_{q}+\frac{h_{q+1}}{2}\right)+F\left(\chi_{q}, t_{q}+\frac{h_{q+1}}{2}\right)\right) \\
\left.+\theta_{3} F\left(\nu_{q}, t_{q}+h_{q+1}\right)\right\}
\end{array}\right.
$$

where

$$
\left\{\begin{array}{l}
\theta_{1}=4 \phi_{3}\left(L h_{q+1}\right)-3 \phi_{2}\left(L h_{q+1}\right)+\phi_{1}\left(L h_{q+1}\right) \\
\theta_{2}=\phi_{2}\left(L h_{q+1}\right)-2 \phi_{3}\left(L h_{q+1}\right) \\
\theta_{3}=4 \phi_{3}\left(L h_{q+1}\right)-\phi_{2}\left(L h_{q+1}\right)
\end{array}\right.
$$

with the functions $\phi_{1}, \phi_{2}$, and $\phi_{3}$ being defined by

$$
\begin{aligned}
& \phi_{1}=\frac{e^{z}-1}{z}, \\
& \phi_{2}=\frac{e^{z}-1-z}{z^{2}}, \\
& \phi_{3}=\frac{e^{z}-1-z-z^{2} / 2}{z^{3}} .
\end{aligned}
$$

Finally, by using inverse Fourier transform, we obtain $u_{j, q}=\mathscr{F}^{-1}\left(\tilde{u}_{k, q}\right)$ is the approximate solution of $u\left(x_{j}, t_{q}\right)$.

For simplicity, the equal step-size is used to discrete time directions. The method presented in this paper allows one to choose different step sizes which are determined by adaptive strategy given in [31]. Next, we proof that the full-discrete scheme (47) satisfies two conservation laws given by Lemma 1.

3.2. The Conservation Laws for ETDRK4. In this subsection, we prove that the scheme given by (47) can keep mass and energy conservation. First, see the following two explanatory notes about ETDRK4.

Note 1. Equation (46) is obtained by integrating

$$
\left(e^{-L t} \tilde{u}_{k}(\cdot, t)\right)=e^{-L t} F\left(\tilde{u}_{k}, t\right)
$$

from $t=t_{q}$ to $t=t_{q+1}$. Then, from the reasoning process of the method ETDRK4 $[29,30]$, we get the following form
TABLE 1: Errors in spatial direction for Example 1.

\begin{tabular}{lccc}
\hline$h$ & $N$ & $\operatorname{Error}\left(l^{\infty}\right)$ & $\operatorname{Order}_{N}$ \\
\hline $1 e-4$ & 6 & $9.6321 e-2$ & - \\
$1 e-4$ & 8 & $6.5321 e-3$ & 9.3539 \\
$1 e-4$ & 10 & $3.4651 e-4$ & 13.1601 \\
$1 e-4$ & 12 & $1.7032 e-5$ & 16.5246 \\
$1 e-4$ & 14 & $7.0652 e-7$ & 20.6454 \\
$1 e-4$ & 16 & $4.1237 e-8$ & 21.2760 \\
$1 e-4$ & 18 & $4.3511 e-9$ & 19.0936 \\
$1 e-4$ & 20 & $5.3511 e-10$ & 19.8909 \\
\hline
\end{tabular}

The errors in $l^{\infty}$ sense vs. various $N$ for Example 1 with $h=10^{-4}$.

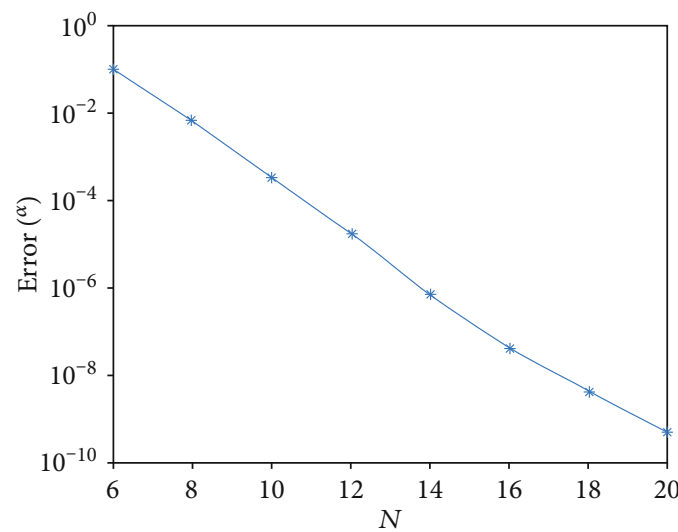

Figure 1: The log-errors (in $l^{\infty}$ sense) vs. $N$ for Example 1.

TABLE 2: Errors in temporal direction for Example 1.

\begin{tabular}{cccc}
\hline$N$ & $h$ & $\operatorname{Error}\left(l^{\infty}\right)$ & Order $_{h}$ \\
\hline 32 & $1 / 100$ & $1.2268 e-5$ & - \\
32 & $1 / 200$ & $8.2231 e-7$ & 3.8991 \\
32 & $1 / 400$ & $6.4230 e-8$ & 3.6784 \\
32 & $1 / 800$ & $4.9085 e-9$ & 3.7099 \\
32 & $1 / 1600$ & $4.6652 e-10$ & 3.3953 \\
32 & $1 / 3200$ & $3.1237 e-11$ & 3.9006 \\
32 & $1 / 6400$ & $2.3511 e-12$ & 3.7318 \\
\hline
\end{tabular}

The errors in $l^{\infty}$ sense vs. different time step size $h$ for Example 1 with $N=32$

$$
e^{-L t_{q+1}} \widehat{u}_{N, k, q+1}=e^{-L t_{q}} \widehat{u}_{N, k, q}+h_{q+1} \Phi\left(t_{q}, \widehat{u}_{N, k, q}\right)+O\left(h_{q+1}^{5}\right)
$$

where

$$
\begin{aligned}
\Phi\left(t_{q}, \widehat{u}_{N, k, q}\right)= & \left.\sum_{j=1}^{4} \frac{h^{j-1}}{j !} \frac{d^{j-1}}{d t^{j-1}}\left(e^{-L t} F\left(\widehat{u}_{N, k, q}, t\right)\right)\right|_{t=t_{q}}, \\
& \widehat{u}_{N, k, q}=\mathscr{F}\left(u_{N}\left(x, t_{q}\right)\right),
\end{aligned}
$$




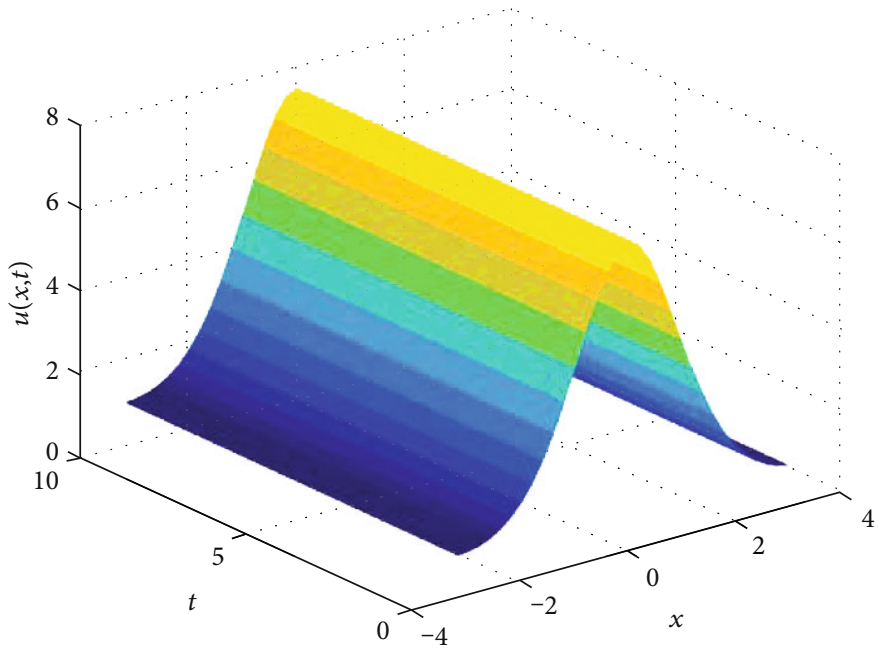

Figure 2: The exact solution on the region $[0,10] \times[-\pi, \pi]$ for Example 1 .

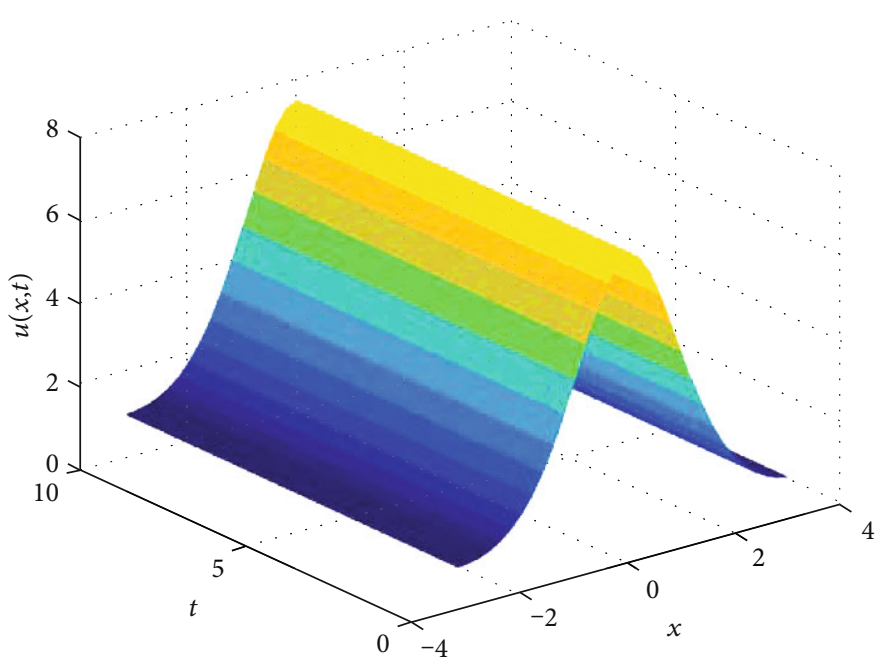

Figure 3: The approximate solution on the region $[0,10] \times[-\pi, \pi]$ for Example 1 .

here, $u_{N}\left(x, t_{q}\right)$ is obtained from the semidiscrete scheme (14). Then, if ignoring the error term $O\left(h_{q+1}^{5}\right)$, using the method of ETDRK4 to solve (46) is equivalent to get the solution to (50) by the scheme (51).

Note 2. Assume that the function $\phi$ mentioned above satisfies the Lipschitz condition with parameter $\lambda>0$, that is

$$
\left|\Phi\left(t, u_{1}\right)-\Phi\left(t, u_{2}\right)\right| \leq \lambda\left|u_{1}-u_{2}\right|
$$

Actually, $\phi$ should be third-order continuous differentiable with respect to $u$ due to using the method of ETDRK4, which ensures that $\phi$ satisfies this assumption easily.

In Lemma 1, we have proved the mass conservation for the semidiscrete scheme (14). Then, if it holds $\mid u_{N}\left(x_{j}, t_{q}\right)-$ $u_{j, q} \mid \leq O\left(h_{\max }^{n}\right)$, the full-discrete scheme ETDRK4 is mass conservative, where $u_{N}\left(x_{j}, t_{q}\right)$ is the solution to (14) and $u_{j, q}$ is obtained from (47) and inverse Fourier transform. Next, we give the proof in the following theorem.

Theorem 5 (mass conservation). Let $u_{N}\left(x_{j}, t_{q}\right)$ be obtained from the semidiscrete scheme (14), $u_{j, q}=\mathscr{F}^{-1}\left(\tilde{u}_{k, q}\right)$, and $\tilde{u}_{k, q}$ is computed by (47). Define the discrete mass $M\left(t_{q}\right)=\sum_{j} \mid \Omega_{j}$ ||$u_{j, q} \mid, \Omega=\bigcup_{j} \Omega_{j}$. Then, ignoring the error comes from machine calculation, we have

$$
\left|u_{N}\left(x_{j}, t_{q}\right)-u_{j, q}\right| \leq O\left(h_{\max }^{4}\right)
$$

where $h_{\max }=\max _{1 \leq i \leq Q} h_{i}, q=0,1,2, \cdots$, Q. Furthermore,

$$
M\left(t_{0}\right) \sim M\left(t_{1}\right) \sim \cdots \sim M\left(t_{Q}\right) .
$$


TABLE 3: The conservation of mass and energy for Example 1.

\begin{tabular}{lccccc}
\hline Conservation & $t=0$ & $t=2$ & $t=6$ & $t=8$ & $t=10$ \\
\hline Mass $\left({ }^{*} 10^{2}\right)$ & 1.058338707 & 1.058338708 & 1.058338710 & 1.058338712 & 1.058338713 \\
Energy $\left({ }^{*} 10^{3}\right)$ & 1.8327299552 & 1.8327299551 & 1.8327299549 & 1.8327299548 & 1.8327299547 \\
\hline
\end{tabular}

The values of mass and energy at time $t=0, t=2, t=6, t=8$, and $t=10$, for Example 1 with $N=16$ and $h=10^{-2}$.

Proof. Without loss of generality, we only need to prove $\mid u_{N}$ $\left(x_{j}, t_{Q}\right)-u_{j, Q} \mid \leq O\left(h_{\max }^{4}\right)$. From Note 1 , we have

$$
e^{-L t_{q+1}} \tilde{u}_{k, q+1}=e^{-L t_{q}} \tilde{u}_{k, q}+h_{q+1} \Phi\left(t_{q}, \tilde{u}_{k, q}\right)
$$

Taking the module of equation (51) minus equation (56), and let $\tilde{e}_{q}=\widehat{u}_{N, k, q}-\tilde{u}_{k, q}$, it follows

$$
e^{-L t_{q+1}}\left|\tilde{e}_{q+1}\right| \leq e^{-L t_{q}}\left|\tilde{e}_{q}\right|+h_{q+1}\left|\Phi\left(t_{q}, \widehat{u}_{N, k, q}\right)-\Phi\left(t_{q}, \tilde{u}_{k, q}\right)\right|+O\left(h_{q+1}^{5}\right) .
$$

Using Note 2, we get

$$
e^{-L t_{q+1}}\left|\tilde{e}_{q+1}\right| \leq e^{-L t_{q}}\left|\tilde{e}_{q}\right|+h_{q+1} \lambda\left|\tilde{e}_{q}\right|+O\left(h_{q+1}^{5}\right),
$$

$q=0,1, \cdots, Q-1$. Summing the above inequalities from $q=0$ to $q=Q-1$, we obtain

$$
e^{-L T}\left|\tilde{e}_{Q}\right| \leq\left|\tilde{e}_{0}\right|+\lambda \sum_{q=0}^{Q-1} h_{q+1}\left|\tilde{e}_{q}\right|+O\left(h_{\max }^{4}\right) .
$$

Let $C_{1}=e^{L T}$ using Gronwall inequality leads to

$$
\left|\tilde{e}_{M}\right| \leq e^{\lambda C_{1}} \sum_{q=0}^{Q-1} h_{q+1}\left(C_{1}\left|\tilde{e}_{0}\right|+O\left(h_{\max }^{4}\right)\right) .
$$

Note that $\tilde{e}_{0}=0$ and denote $e^{\lambda C_{1} T}$ by $C$, the desired result follows,

$$
\left|u_{N}\left(x_{j}, t_{Q}\right)-u_{j, Q}\right|=\left|\mathscr{F}^{-1}\left(\tilde{e}_{Q}\right)\right|=\left|\left(\tilde{e}_{Q}\right)\right| \leq C \cdot O\left(h_{\max }^{4}\right)=O\left(h_{\max }^{4}\right) .
$$

Moreover, we have

$$
\begin{aligned}
\left|\left(\left|u_{j, 0}\right|-\left|u_{j, Q}\right|\right)\right| & \leq\left|\left(\left|u_{j, 0}\right|-\left|u_{N}\left(x_{j}, t_{0}\right)\right|+\left|u_{N}\left(x_{j}, t_{Q}\right)\right|-\left|u_{j, Q}\right|\right)\right| \\
& \leq\left|\left(\left|u_{j, 0}\right|-\left|u_{N}\left(x_{j}, t_{0}\right)\right|\right)\right|+\left|\left(\left|u_{N}\left(x_{j}, t_{Q}\right)\right|-\left|u_{j, Q}\right|\right)\right| \\
& \leq 2 O\left(h_{\max }^{4}\right)=O\left(h_{\max }^{4}\right) .
\end{aligned}
$$

The above inequalities imply

$$
M(0) \sim M\left(t_{1}\right) \sim \cdots \sim M\left(t_{Q}\right),
$$

as $N$ is big enough and $h_{\max } \longrightarrow 0$.
TABLE 4: Errors in temporal direction for Example 2.

\begin{tabular}{lccc}
\hline$N$ & $h$ & $\operatorname{Error}\left(l^{\infty}\right)$ & Order $_{h}$ \\
\hline 32 & $1 / 50$ & $8.6535 e-07$ & - \\
32 & $1 / 100$ & $5.4832 e-08$ & 3.9802 \\
32 & $1 / 200$ & $4.1220 e-09$ & 3.7336 \\
32 & $1 / 400$ & $3.1075 e-10$ & 3.7295 \\
32 & $1 / 800$ & $2.2452 e-11$ & 3.7908 \\
32 & $1 / 1600$ & $1.5247 e-12$ & 3.8802 \\
32 & $1 / 3200$ & $1.0521 e-13$ & 3.8572 \\
32 & $1 / 6400$ & $1.0012 e-14$ & 3.3935 \\
\hline
\end{tabular}

The errors in $l^{\infty}$ sense vs. different time step size $h$ for Example 2 with $N=32$

Theorem 6 (energy conservation). Let

$$
u_{j, q}=\mathscr{F}^{-1}\left(\tilde{u}_{k, q}\right)
$$

and $\tilde{u}_{k, q}$ is obtained from (47). Define the discrete local-energy

$$
E_{j}\left(t_{q}\right)=\left|\Omega_{j}\right|\left\{-\alpha\left(\bar{u}_{j, q} \delta^{\alpha / 2} u_{j, q}\right)+\frac{\beta}{2}\left|u_{j, q}\right|^{4}\right\},
$$

and the discrete energy

$$
E\left(t_{q}\right)=\sum_{j} E_{j}\left(t_{q}\right)
$$

here, $\delta^{\alpha / 2} u_{j, q}$ is a numerical approximation of the fractional Laplacian operator $(-\Delta)^{\alpha / 2}, \Omega=\bigcup_{j} \Omega_{j}$. Then, we have

$$
\left|E_{j}(0)-E_{j}\left(t_{q}\right)\right| \leq O\left(h_{\max }^{4}\right), q=1,2, \cdots, Q .
$$

Furthermore, $E\left(t_{0}\right) \sim E\left(t_{1}\right) \sim \cdots \sim E\left(t_{Q}\right)$.

Proof. Without loss of generality, we only need to prove

$$
\left|E_{j}(0)-E_{j}\left(t_{Q}\right)\right| \leq O\left(h_{\max }^{4}\right) .
$$


TABLE 5: The conservation of mass and energy for Example 2.

\begin{tabular}{lccccc}
\hline Conservation & $t=0$ & $t=10$ & $t=20$ & $t=30$ & $t=50$ \\
\hline Mass & 3.1415926535 & 3.1415926536 & 3.1415926536 & 3.1415926536 & 3.1415926537 \\
Energy & 1.178097245 & 1.178097245 & 1.178097245 & 1.178097245 & 1.178097245 \\
\hline
\end{tabular}

The values of mass and energy at time $t=0, t=10, t=20, t=30$, and $t=50$, for Example 2 with $N=16$ and $h=10^{-2}$.

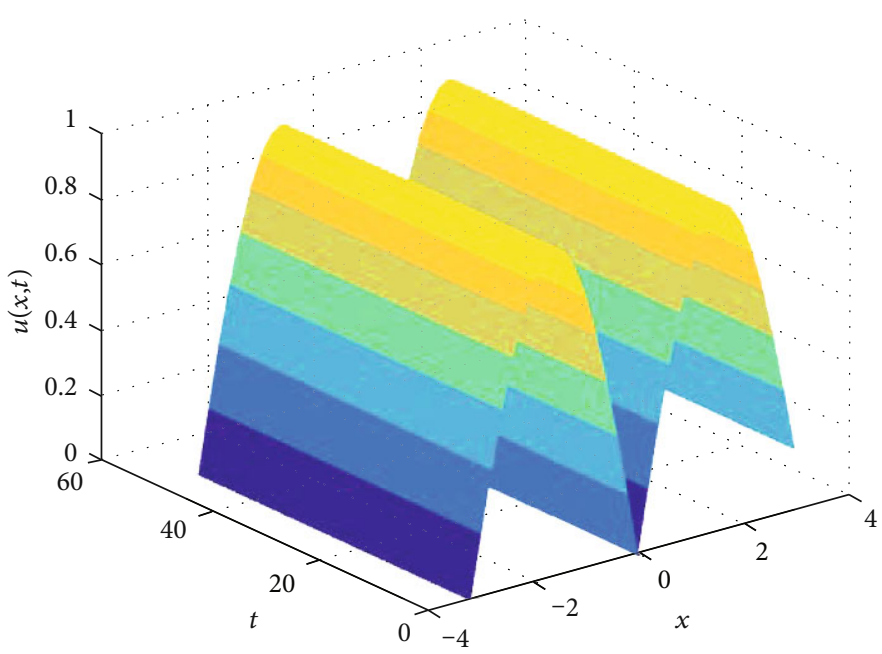

FIgURE 4: The exact solution on the region $[0,60] \times[-\pi, \pi]$ for Example 2.

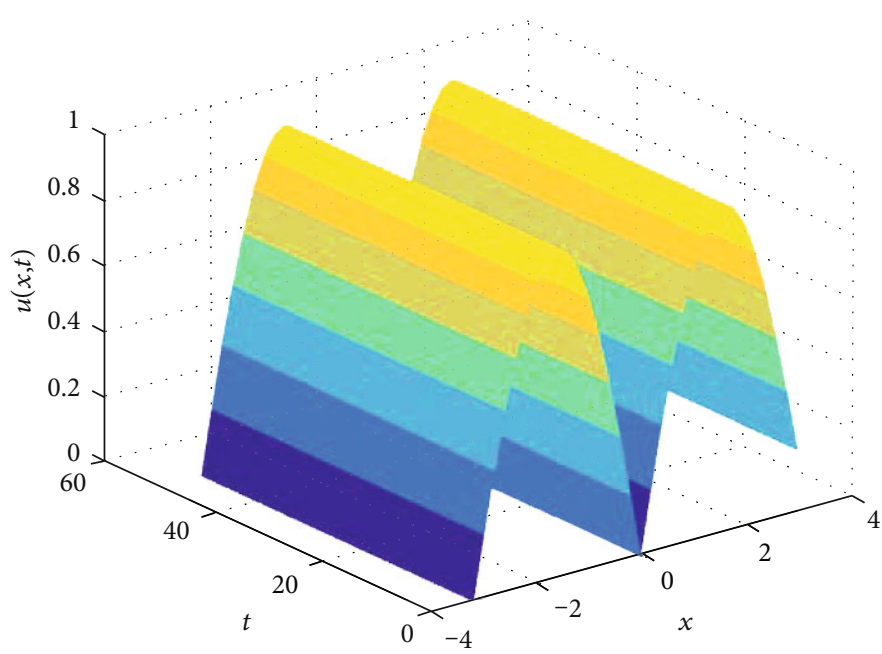

FIGURE 5: The approximate solution on the region $[0,60] \times[-\pi, \pi]$ for Example 2 .

Define bounded linear operator

$$
A\left(\left|u_{j, q}\right|\right):=E_{j}\left(t_{q}\right)
$$

then we have

$$
\left|E_{j}(0)-E_{j}\left(t_{Q}\right)\right|=\left\|A\left(\left|u_{j, 0}\right|-\left|u_{j, Q}\right|\right)\right\| \leq C\left|\left(\left|u_{j, 0}\right|-\left|u_{j, Q}\right|\right)\right|,
$$

where $C>0$ is a positive constant. By Theorem 5 ,

$$
\left|E_{j}(0)-E_{j}\left(t_{Q}\right)\right| \leq O\left(h_{\max }^{4}\right)
$$

which implies

$$
E(0) \sim E(1) \sim \cdots \sim E(Q)
$$

as $N$ is big enough and $h_{\max } \longrightarrow 0$.

\section{Applying}

In this section, we present two examples to illustrate our results. As in [10], we define the convergence orders in $\left(l^{\infty}\right)$ norm sense as follows 


$$
\begin{aligned}
\operatorname{order}_{h} & =\frac{\log \left(E\left(h_{1}, N\right) / E\left(h_{2}, N\right)\right)}{\log \left(h_{1} / h_{2}\right)}, \\
\operatorname{order}_{N} & =\frac{\log \left(E\left(h, N_{1}\right) / E\left(h, N_{2}\right)\right)}{\log \left(N_{2} / N_{1}\right)},
\end{aligned}
$$

where $E(h, N)$ is given by

$$
E(h, N)=\max _{j, p}\left|u\left(x_{j}, t_{p}\right)-u_{j, p}\right| \cdot
$$

All the experiments were done on a computer with the following configuration: Intel(R) Core (TM) i5-8250 CPU @ $1.60 \mathrm{GHz} 1.80 \mathrm{GHz}$ and involved using MATLAB 2015b.

4.1. Example 1. We first consider the periodic initial Schrödinger equation (1) with the following conditions: $0 \leq t \leq 10$ , $\quad \alpha=1, \beta=-1, \gamma=-1, \quad f(x)=\sin ^{2}(x)-\cos (x)-$ $(\exp (\cos (x)+1))^{2}+1$, and $u_{0}(x)=\exp (\cos (x)+1)$. The exact solution is

$$
u(x, t)=\exp (\cos (x)+1) \exp (-i t)
$$

By FFT and ETDRK4, we compute numerical solution of this example. The point-wise errors (in $l_{\infty}$ sense) against various $N$ are shown in Table 1 ; then, we plot in semilog scale in Figure 1, which clearly indicates the exponential convergence in space direction, and the fourth order accuracy in time direction is shown in Table 2. The exact solution is shown in Figure 2, and the solution surface is shown in Figure 3, from which we can see that the two pictures are fit very well. Finally, we compute the mass and the energy of the numerical solution at different times. As one can see in Table 3, the scheme has an excellent conservation property and is in well agreement with the results in Theorem 5.

4.2. Example 2. We then consider the periodic initial Schrödinger equation (1) with the following conditions: $0 \leq t \leq 50$ , $\alpha=1, \beta=-1, \gamma=-1, f(x)=\cos ^{2}(x)$, and $u_{0}(x)=\sin (x)$. The exact solution to the equation is

$$
u(x, t)=\sin (x) \exp (i(-1.5 t))
$$

We compute the numerical solution for this example until the time $t=50$ with time step $h=0.01$ on the uniform grid with $N=32$. We display the errors that derived from temporal direction in Table 4. By Theorem 5, the scheme can keep the mass and energy conservation. Table 5 validates this result very well. It can be seen from Figures 4 and 5 that the approximate solution is in good agreement with the exact solution.

\section{Conclusion}

In this paper, we propose a method to solve the Schrödinger equations by using the method of Fourier spectral and modified fourth order exponential time-differencing RungeKutta, and we give the semidiscrete and full-discrete schemes for the equations. We prove that the schemes can keep the conservation law of mass and energy for full-discrete schemes. Numerical examples verify that our conclusions are correct.

\section{Data Availability}

The numerical data used to support the findings of this study are included within the article.

\section{Conflicts of Interest}

The authors declare that there is no conflict of interests regarding the publication of this paper.

\section{Acknowledgments}

The authors thank the referees for their many constructive comments and suggestions to improve the paper. This work was partly supported by the Educational science project of Guangdong Province with Nos. 2020GXJK385 and 2019KTSCX097.

\section{References}

[1] N. Laskin, "Fractional quantum mechanics," Physical Review E, vol. 62, no. 3, pp. 3135-3145, 2000.

[2] S. Z. Rida, H. M. El-Sherbiny, and A. A. M. Arafa, "On the solution of the fractional nonlinear Schrodinger equation," Physics Letters A, vol. 372, no. 5, pp. 553-558, 2008.

[3] J. Liu and G. Hou, "Numerical solutions of the space- and time-fractional coupled Burgers equations by generalized differential transform method," Applied Mathematics and Computation, vol. 217, no. 16, pp. 7001-7008, 2011.

[4] L. Wei, Y. He, X. Zhang, and S. Wang, "Analysis of an implicit fully discrete local discontinuous Galerkin method for the time-fractional Schrödinger equation," Finite Elements in Analysis and Design, vol. 59, pp. 28-34, 2012.

[5] S. Secchi, "Ground state solutions for nonlinear fractional Schrödinger equations in RN," Journal of Mathematical Physics, vol. 54, no. 3, article 031501, 2013.

[6] A. H. Bhrawy, E. H. Doha, S. S. Ezz-Eldien, and R. A. Van Gorder, "A new Jacobi spectral collocation method for solving 1 +1 fractional Schrödinger equations and fractional coupled Schrödinger systems," The European Physical Journal Plus, vol. 129, no. 12, p. 260, 2014.

[7] D. Wang, A. Xiao, and W. Yang, "A linearly implicit conservative difference scheme for the space fractional coupled nonlinear Schrödinger equations," Journal of Computational Physics, vol. 272, pp. 644-655, 2014.

[8] P. Wang and C. Huang, "An energy conservative difference scheme for the nonlinear fractional Schrödinger equations," Journal of Computational Physics, vol. 293, pp. 238-251, 2015.

[9] M. Ran and C. Zhang, "A conservative difference scheme for solving the strongly coupled nonlinear fractional Schrödinger equations," Communications in Nonlinear Science and Numerical Simulation, vol. 41, pp. 64-83, 2016.

[10] M. Li, C. Huang, and P. Wang, "Galerkin finite element method for nonlinear fractional Schrödinger equations," Numerical Algorithms, vol. 74, no. 2, pp. 499-525, 2017.

[11] M. Li, X. M. Gu, C. Huang, M. Fei, and G. Zhang, "A fast linearized conservative finite element method for the strongly 
coupled nonlinear fractional Schrödinger equations," Journal of Computational Physics, vol. 358, pp. 256-282, 2018.

[12] M. Li, C. Huang, and W. Ming, "A relaxation-type Galerkin FEM for nonlinear fractional Schrödinger equations," Numerical Algorithms, vol. 83, no. 1, pp. 99-124, 2020.

[13] Z. Q. Wang, J. F. Yin, and Q. Y. Dou, "Preconditioned modified Hermitian and skew-Hermitian splitting iteration methods for fractional nonlinear Schrodinger equations," Journal of Computational and Applied Mathematics, vol. 367, article 112420, 2020.

[14] Y. Yang, J. Wang, S. Zhang, and E. Tohidi, "Convergence analysis of space-time Jacobi spectral collocation method for solving time-fractional Schrodinger equations," Applied Mathematics and Computation, vol. 387, article 124489, 2020.

[15] S. Liang, D. D. Repovš, and B. Zhang, "Fractional magnetic Schrödinger-Kirchhoff problems with convolution and critical nonlinearities," Mathematical Methods in the Applied Sciences, vol. 43, no. 5, pp. 2473-2490, 2020.

[16] A. D. Ionescu and F. Pusateri, "Nonlinear fractional Schrödinger equations in one dimension," Journal of Functional Analysis, vol. 266, no. 1, pp. 139-176, 2014.

[17] M. Edwards and K. Burnett, "Numerical solution of the nonlinear Schrödinger equation for small samples of trapped neutral atoms," Physical Review A, vol. 51, no. 2, article 1382, 1386 pages, 1995.

[18] Q. Chang, E. Jia, and W. Sun, "Difference schemes for solving the generalized nonlinear Schrödinger equation," Journal of Computational Physics, vol. 148, no. 2, pp. 397-415, 1999.

[19] W. Bao, S. Jin, and P. A. Markowich, "Numerical study of time-splitting spectral discretizations of nonlinear Schrödinger equations in the semiclassical regimes," SIAM Journal on Scientific Computing, vol. 25, no. 1, pp. 27-64, 2003.

[20] H. Wang, "Numerical studies on the split-step finite difference method for nonlinear Schrödinger equations," Applied Mathematics and Computation, vol. 170, no. 1, pp. 17-35, 2005.

[21] M. Dehghan and V. Mohammadi, "A numerical scheme based on radial basis function finite difference (RBF-FD) technique for solving the high-dimensional nonlinear Schrödinger equations using an explicit time discretization: Runge-Kutta method," Computer Physics Communications, vol. 217, pp. 23-34, 2017.

[22] M. Ahsan, I. Ahmad, M. Ahmad, and I. Hussian, "A numerical Haar wavelet-finite difference hybrid method for linear and non-linear Schrödinger equation," Mathematics and Computers in Simulation, vol. 165, pp. 13-25, 2019.

[23] L. Banjai and M. López-Fernández, "Numerical approximation of the Schrodinger equation with concentrated potential," Journal of Computational Physics, vol. 405, article 109155, 2020.

[24] S. Jin, L. Liu, G. Russo, and Z. Zhou, "Gaussian wave packet transform based numerical scheme for the semi-classical Schrodinger equation with random inputs," Journal of Computational Physics, vol. 401, article 109015, 2020.

[25] J. Wang and A. Xiao, "Conservative Fourier spectral method and numerical investigation of space fractional KleinGordon-Schrodinger equations," Applied Mathematics and Computation, vol. 350, pp. 348-365, 2019.

[26] B. E. Moore, L. Noreña, and C. M. Schober, "Conformal conservation laws and geometric integration for damped Hamiltonian PDEs," Journal of Computational Physics, vol. 232, no. 1, pp. 214-233, 2013.
[27] S. R. K. Iyengar, G. Jayaraman, and V. Balasubramanian, "Variable mesh difference schemes for solving a nonlinear Schrödinger equation with a linear damping term," Computers \& Mathematcs with Applications, vol. 40, no. 12, pp. 1375$1385,2000$.

[28] W. P. Hu, Z. C. Deng, and T. T. Yin, "Almost structurepreserving analysis for weakly linear damping nonlinear Schrödinger equation with periodic perturbation," Communications in Nonlinear Science and Numerical Simulation, vol. 42, pp. 298-312, 2017.

[29] S. M. Cox and P. C. Matthews, "Exponential time differencing for stiff systems," Journal of Computational Physics, vol. 176, no. 2, pp. 430-455, 2002.

[30] A. Kassam and L. N. Trefethen, "Fourth-order time-stepping for stiff PDEs," SIAM Journal on Scientific Computing, vol. 26, no. 4, pp. 1214-1233, 2005.

[31] H. Qu and Z. She, "Fourier spectral method with an adaptive time strategy for nonlinear fractional Schrödinger equation," Numerical Methods for Partial Differential Equations, vol. 36, no. 4, pp. 823-838, 2020. 